\title{
La posición de la hoja y su efecto sobre la calidad y producción de frutos de vid (Vitis vinifera L.) var. Riesling $\mathrm{x}$ Silvaner
}

\section{Leaf position and its effect on quality and yield of the grapevine fruit (Vitis vinifera L.) 'Riesling x Silvaner'}
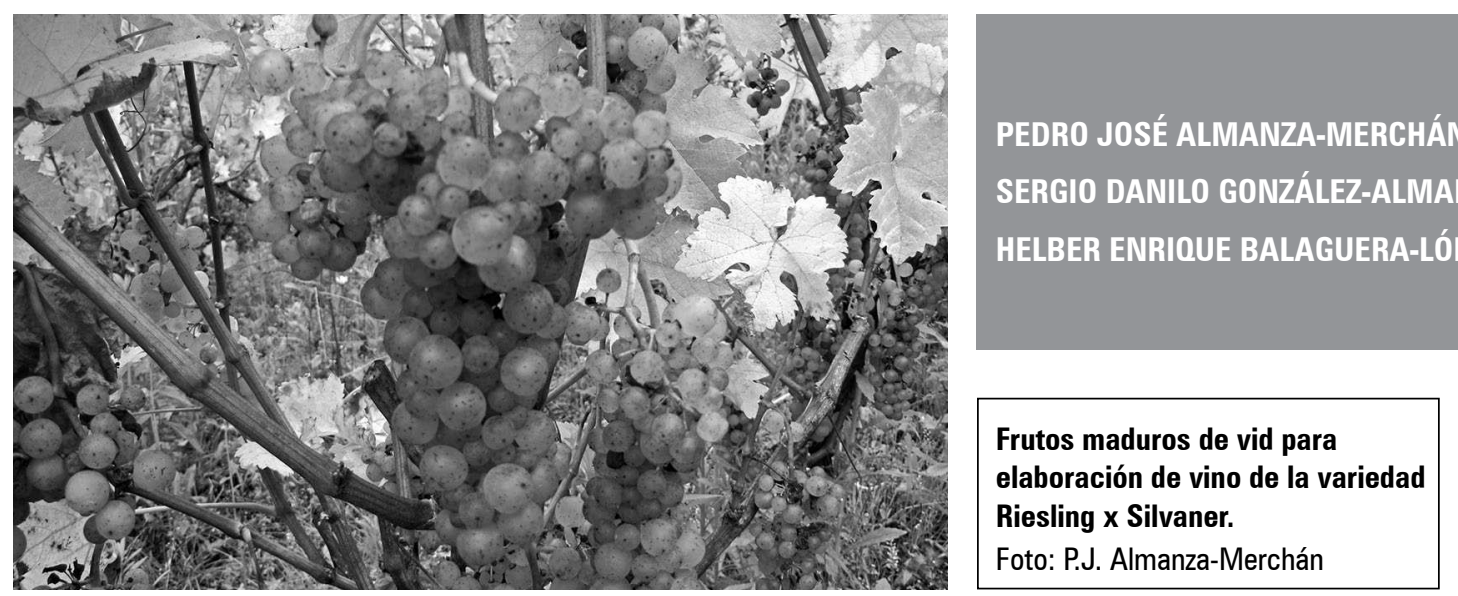

\section{RESUMEN}

En Boyacá, durante los últimos 29 años se viene cultivando uva para elaboración de vino. El desarrollo de las hojas durante el crecimiento es fundamental para la interceptación de luz, el proceso fotosintético y el control de la producción de materia seca. Sin embargo, son las responsables de la generación de sombra en las plantas, lo cual puede disminuir el rendimiento y la calidad de bayas. El objetivo de esta investigación fue evaluar el efecto de la posición de la hoja de plantas de vid (Vitis vinifera L.) 'Riesling x Silvaner' sobre componentes de calidad y producción de las bayas. Se utilizó un diseño experimental completamente al azar con arreglo factorial de $4 \times 3$, el primer factor correspondió a los estadios fenológicos del fruto (herbáceo, envero, maduración y vendimia) y el segundo, a la localización de la hoja en el pámpano (hojas 4, 5 y 6). Los resultados indicaron que los sólidos solubles totales (SST) aumentaron con el desarrollo del fruto, siendo mayores en la vendimia, en este mismo estado, la hoja 6 favoreció mayor acumulación de SST. Las masas fresca y seca del fruto fueron mayores a medida que avanzó el estado de desarrollo de las bayas, se observó que la hoja 6 en cualquiera de los estados de desarrollo generó mayor masa fresca de bayas, mientras que en la masa seca, la posición de la hoja no tuvo efecto significativo. No obstante, se considera que la hoja 6 tiene un efecto favorable sobre la producción y calidad de frutos de uva 'Riesling x Silvaner'

Facultad de Ciencias Agropecuarias, Grupo de Investigaciones Ecofisiología Vegetal, Universidad Pedagógica y Tecnológica de Colombia, Tunja (Colombia).

2 Facultad de Ciencias Agropecuarias, Grupo de Investigaciones Agrícolas, Universidad Pedagógica y Tecnológica de Colombia, Tunja (Colombia).

3 Programa de Doctorado en Ciencias Agropecuarias, Área Agraria con Énfasis en Fisiología de Cultivos, Facultad de Agronomía, Universidad Nacional de Colombia, Bogotá; Facultad de Ciencias Agropecuarias, Grupo de Investigaciones Agrícolas, Universidad Pedagógica y Tecnológica de Colombia, Tunja (Colombia).

4 Autor para correspondencia.ppcalma@gmail.com 


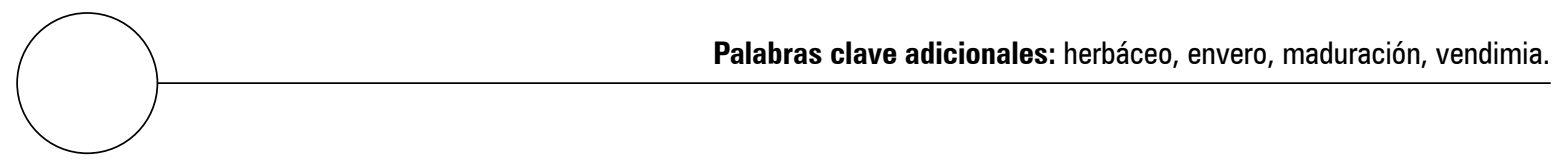

\section{ABSTRACT}

In Boyacá, grapes have been grown for winemaking for the past 29 years. The development of the leaves during growth is essential for the interception of light, the photosynthetic process and the control of the yield of dry matter. However, leaves are also responsible for the generation of shadow within the plants, which can decrease yield and quality of the berries. The objective of this research was to evaluate the effect of leaf position of grapevines (Vitis vinifera L.) 'Riesling x Silvaner', on quality components and berry yield. A completely randomized design was used with a factorial arrangement of $4 \times 3$; the first factor corresponded to the phenological stages of the fruit (herbaceous, veraison, maturation and harvest) and the second to the location of the leaf on the branch (leaves 4, 5 and 6). The results showed that total soluble solids (TSS) increased with fruit development, being higher at harvest; in this stage, leaf 6 favored greater accumulation of TSS. The fresh and dry mass of the fruit were higher as the state of development of the berries advanced; it was observed that leaf 6 in any state of development resulted in greater fresh weight of the berries, while for dry mass, the leaf position had no significant effect. As a result, leaf 6 is considered as having a favorable effect on yield and fruit quality of the grape 'Riesling x Silvaner'.

Additional key words: herbaceous, veraison, maturation, harvest.

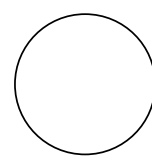

En 16 municipios de dos regiones ubicadas entre los $2.100 \mathrm{msnm}$ (Ricaurte alto) a $2.650 \mathrm{msnm}$ (Sugamuxi, Valderrama, Tundama, Norte y Gutiérrez), en el departamento de Boyacá durante los últimos 29 años, se viene cultivando uva para elaboración de vino (Almanza, 2011), a pesar de ser una planta originaria de la zona templada del Asia occidental, bajo las condiciones agroecológicas de las zonas de clima frío tropical, las plantas se han adaptado presentando rendimientos de 5,53 t ha-1 (Agronet, 2012), proporcionando frutos que originan vinos de alta calidad.

La radiación solar es la fuente primaria de energía usada por las plantas para desarrollar el proceso de la fotosíntesis. Mediante este proceso se producen los elementos necesarios para que los organismos vegetales cumplan con su función básica de crecimiento y desarrollo. Parte de la materia vegetal producida da como resultado el producto a ser cosechado (Hernández et al., 2001). De esta manera, Rajapakse y Shahak (2007), mencionan que las características de la luz tiene un efecto determinante en la morfogénesis vegetal y sus efectos se utilizan en beneficio de los agricultores que buscan mayores calidades del fruto $y$, por ende, producciones controladas. Por ello, la mayoría de los viñedos destinados para elaboración de vinos se sitúan en zonas con características agroclimáticas en donde se presenta alta luminosidad y periodos de baja precipitación, especialmente durante la época de maduración del fruto (Almanza, 2012).

La cantidad de área foliar que desarrolla la planta de uva, es un factor que determina la capacidad 
productiva, por ser la responsable de la actividad fotosintética (Sánchez de Miguel, 2007). La disposición de la cubierta vegetal y su densidad, determinan la interceptación y distribución de la radiación por la planta (Zufferey et al., 2000; Schultz 1995), y por tanto el microclima luminoso de las hojas (Katerji et al., 1994) que condicionará la fotosíntesis (Schultz, 1995) y la transpiración (Mabrouk et al., 1997), y como consecuencia la productividad y la calidad de la cosecha (Hidalgo, 1999). Por lo cual, según Hunter y Archer (2002), la importancia de la función del follaje a través de la vida de las plantas es una práctica integral esencial en viticultura y enología en busca de la obtención y el mejoramiento de la calidad de la uva y el vino (Kliewer, 1980; Hunter, 2000).

El desarrollo de las hojas durante el crecimiento es fundamental para la interceptación de luz, el proceso fotosintético y el control de la producción de materia seca (Oliveira y Santos, 1995). Sin embargo, las hojas también son las principales responsables de la generación de sombra dentro de las plantas, lo cual puede causar una disminución en el rendimiento y en la calidad de bayas (Smart y Smith, 1988). La posición que ocupan las diferentes estructuras de la planta (nudo, entrenudo, yema y hoja), puede afectar al patrón de desarrollo como es el caso de manzano (Johnson y Lakso, 1985) y kiwi (Seleznyova y Greer, 2001). En la vid, las hojas que nacen en la parte basal de los pámpanos se extienden más rápidamente que las terminales. Este comportamiento, por la posición, es un efecto de tipo genético más que por las condiciones climáticas, sin embargo, en plantas perennes, el crecimiento inicial de los brotes está fuertemente influenciado por las reservas acumuladas durante el crecimiento anterior (Sánchez de Miguel, 2007).

Hunter et al. (1995) encontraron que en los primeros estadios de crecimiento del fruto en Vitis vinifera, son las hojas basales y las opuestas al racimo las principales fuentes de fotoasimilados para los racimos de su propio pámpano. En tanto que después de la parada de crecimiento, que coincide con el envero, todas las hojas situadas en el mismo pámpano, suministran los asimilados que sus racimos demandan (Stewart et al., 1996). Los brotes sin racimos dirigen sus asimilados, casi en su totalidad, hacia los racimos ubicados en brotes fértiles. Por ello, Sánchez de Miguel (2007) menciona que es importante caracterizar la superficie foliar desarrollada por el pámpano de tipo medio, que es la principal fuente de asimilados de los racimos de dicho pámpano, y cómo se distribuye en toda su longitud debido a los movimientos de asimilados a lo largo del pámpano, siendo importante describir las hojas del canopy en función de su edad, ya que esta influye decisivamente en su actividad fotosintética y, por tanto, en la productividad global de la cepa.

Las hojas de la vid se comportan como fuentes de carbohidratos hasta que alcanzan entre $50 \%$ y $80 \%$ de su tamaño final (Koblet, 1969). La mayor eficiencia fotosintética foliar se produce en hojas recientemente expandidas, ya que tras alcanzar el tamaño foliar final (aproximadamente 40 d tras la aparición) empieza a descender (Alleweldt et al., 1982; Vasconcelos y Castagnoli, 2000). Buttrose (1966) encontró que los pámpanos dejan de ser vertederos cuando el área foliar del pámpano alcanzó los 50 cm² . Candolfi-Vasconcelos et al. (1994) encontraron que, durante el periodo de maduración, las hojas más eficientes son las principales que se encuentran en la parte superior del canopy y las hojas insertadas sobre los brotes que se desarrollan durante el mismo periodo de crecimiento de los pámpanos.

En la región productora de Boyacá, no se han realizado investigaciones que conduzcan a determinar cuál es la relación de las hojas próximas al racimo y su posible efecto en la calidad del fruto y su implicación en las propiedades del vino. Por tanto, el objetivo de esta investigación fue evaluar el efecto de la posición de la hoja de plantas de vid de la selección clonal 'Riesling x Silvaner' sobre los componentes de calidad y producción 
de frutos bajo las condiciones agroecológicas de Corrales, Boyacá.

\section{MATERIALES Y MÉTODOS}

La investigación en la fase de campo se realizó durante el segundo semestre del año 2011 y parte del primer semestre de 2012 en el viñedo del municipio de Corrales, Boyacá, situado a 546'47,1" $\mathrm{N}$ y $72^{\circ} 58^{\prime} 36,5^{\prime \prime} \mathrm{W}$, a una altitud de $2.450 \mathrm{~m}$. El clima de la zona se caracteriza por presentar una temperatura promedio anual de $16,5^{\circ} \mathrm{C}$ y precipitación bimodal media de $700 \mathrm{~mm}$ año-1 con dos picos ubicados entre abril-mayo y octubrenoviembre. Los suelos son de textura liviana, del tipo franco-arenoso y fertilidad natural baja, de acuerdo con el sistema de clasificación de zonas de vida propuestas por Holdrige, se presenta una sola zona de vida: bosque seco montano bajo (Bs-MB). Los suelos son pedregosos de textura liviana, de tipo franco-arenoso y fertilidad natural baja (Almanza, 2011).

El comportamiento del clima durante el experimento, fue atípico, debido a que se presentó el fenómeno de la niña. Los datos climatológicos, se reportan en la tabla 1, la temperatura promedio fue de $14,73^{\circ} \mathrm{C}$, la temperatura máxima y mínima promedio fueron de 20,07 y $9,82^{\circ} \mathrm{C}$, respectivamente, la humedad relativa fue del $86,31 \%$ y precipitación acumulada de $670,3 \mathrm{~mm}$, que correspondió al triple de lo normal. Se presentó un promedio de $4,36 \mathrm{~h} \mathrm{~d}^{-1}$ de brillo solar.

Las plantas objeto de estudio fueron de la selección clonal de Vitis vinifera L. variedad Riesling x Silvaner, también conocida como Riesling Becker (Almanza, 2012) procedente de Alsacia, Francia en la frontera con Alemania; de la cual se elabora vino de reconocida calidad (Quijano, 2001). Las plantas tienen una edad de 9 años y están sembradas a distancia de 1,20 $\times 0,90 \mathrm{~m}$, el sistema de manejo es el de tipo guyot simple con conducción en espaldera a tres alambres.

Cuando las plantas llegaron al estadio principal 5 , de la escala $(\mathrm{BBCH})$ propuesta por Lorenz et al. (1995), correspondiente a racimos florales visibles, se marcaron, en cada una de ellas, las hojas 4, 5 y 6 , y se realizaron las evaluaciones cuando alcanzaron los estadios 75, 80, 85 y 89 (herbáceo, envero, maduración y vendimia). La determinación del comportamiento de componentes de producción y calidad durante el crecimiento y desarrollo, se realizó en el laboratorio de fisiología vegetal de la Universidad Pedagógica y Tecnológica de Colombia en Tunja. Los sólidos

Tabla 1. Variables ambientales de Corrales (Boyacá, Colombia) entre los meses de julio a diciembre de 2011.

\begin{tabular}{|l|c|c|c|c|c|c|}
\hline \multicolumn{1}{|c|}{ Mes } & $\begin{array}{c}\text { Precipitación } \\
(\mathrm{mm})\end{array}$ & $\begin{array}{c}\text { Temperatura } \\
\text { media }\left({ }^{\circ} \mathrm{C}\right)\end{array}$ & $\begin{array}{c}\text { Temperatura } \\
\text { máxima }\left({ }^{\circ} \mathrm{C}\right)\end{array}$ & $\begin{array}{c}\text { Temperatura } \\
\text { mínima }\left({ }^{\circ} \mathrm{C}\right)\end{array}$ & $\begin{array}{c}\text { Brillo solar } \\
\left(\mathrm{h} \mathrm{d}^{-1}\right)\end{array}$ & $\begin{array}{c}\text { Humedad } \\
\text { relativa }(\%)\end{array}$ \\
\hline Mayo & 169,6 & 12,7 & 19,6 & 8,5 & 3,8 & 90,4 \\
\hline Junio & 30,2 & 16,8 & 20,2 & 10,7 & 5,4 & 85,5 \\
\hline Julio & 31,2 & 16,6 & 21,6 & 10 & 5,5 & 86,2 \\
\hline Agosto & 61,4 & 15,1 & 20,5 & 10,8 & 5,5 & 87,8 \\
\hline Septiembre & 44,6 & 15,3 & 19,1 & 10,4 & 5,3 & 84,4 \\
\hline Octubre & 181,9 & 12,6 & 18,6 & 8,4 & 2,3 & 91,6 \\
\hline Noviembre & 139,9 & 12,7 & 18,4 & 9,8 & 2,2 & 90,0 \\
\hline Diciembre & 11,5 & 16,1 & 22,6 & 10 & 4,9 & 74,6 \\
\hline Promedio & $670,3^{*}$ & 14,73 & 20,07 & 9.82 & 4,36 & 86,31 \\
\hline
\end{tabular}

*El valor de la precipitación es el acumulado durante el periodo del estudio. Fuente: Almanza, 2012. 
solubles totales (SST) se determinaron a través de mediciones de grados Brix, con un refractómetro manual Atago de rango 0 a $32 \%$ a $20^{\circ} \mathrm{C}$. La masa fresca se evaluó tomando 10 frutos que se pesaron con una balanza de precisión Acculab Sartorius group $(0,001 \mathrm{~g}$ de exactitud). La masa seca se determinó después de secar los 10 frutos en un horno Memmert UB-500, a temperatura de $85^{\circ} \mathrm{C}$ hasta peso constante $(48 \mathrm{~h})$.

Se empleó un diseño experimental completamente al azar con arreglo factorial de $4 \times 3$. Donde el primer factor correspondió a los estadios fenológicos del fruto (herbáceo, envero, maduración y vendimia) y el segundo, a la localización de la hoja en el pámpano (hojas 4, 5 y 6), para un total de 12 tratamientos. Cada tratamiento estuvo conformado por 5 repeticiones para un total de 60 unidades experimentales (UE), cada UE estuvo compuesta por dos plantas. Los resultados se sometieron a análisis de varianza factorial y se llevó a cabo la prueba de comparación múltiple de Tukey $(P \leq 0,05)$. Para el análisis de los datos se utilizó el programa estadístico SAS® v. 9.2 .

\section{RESULTADOS Y DISCUSIÓN}

\section{Sólidos solubles totales}

La ubicación de la hoja no generó diferencias estadísticas en los sólidos solubles totales de los frutos uva variedad Riesling x Silvaner, pero sí lo hizo el estado fenológico, donde el valor más alto se obtuvo en la vendimia con $22,1^{\circ}$ Brix (figura $1 \mathrm{~B})$. Los tratamientos también generaron efecto significativo $(P \leq 0,01)$, el mayor contenido de SST se obtuvo en los frutos ubicados en el costado opuesto a la hoja 6 , durante la vendimia con un valor de $22,98^{\circ} \mathrm{Brix}$, mientras que las bayas ubicadas a la altura de la hoja 5 presentaron SST de 21,66 ${ }^{\circ}$ Brix (figura $1 \mathrm{~A}$ ).

Los sólidos solubles totales en uva para elaboración de vino son uno de los principales com- puestos que garantizan la calidad de los vinos. Por ello, se ha considerado como el principal factor regulador del precio del fruto de uva (Almanza, 2011). La curva típica de crecimiento doble sigmoidal muestra un incremento lento durante el estadio herbáceo, aumentando rápidamente después del envero desde 12,68 ${ }^{\circ}$ Brix hasta llegar al máximo en el momento de la recolección del fruto. Diferentes autores (Conde et al., 2007; Alí et al., 2011) mencionan que la causa de este incremento se debe a que durante la maduración los azúcares de mayor concentración en el fruto corresponden a glucosa y fructuosa, a lo que se suma la alta actividad de la enzima invertasa ácida (Fillion et al., 1999).

En la figura 1A, se observa que en la vendimia, el mayor contenido de SST fue generado por la hoja 6 , seguido de la 5 , y el menor aporte lo hizo la hoja 4; precisamente, en la variedad evaluada genotípicamente, los racimos se ubican frente a las hojas quinta y sexta. En la investigación, queda demostrado lo expuesto por Motomura (1990), quien afirma que las hojas que aportan en mayor cuantía con fotoasimilados al racimo es la opuesta, debido a que la sacarosa sigue caminos determinados por los ortósticos o filotaxis dística, las hojas que están en el mismo ortóstico que los racimos son las primeras que fomentan la descarga de asimilados, hasta envero. La calidad de las bayas aumenta debido a que el proceso fotosintético responsable de la exportación de sacarosa a las bayas, determina la capacidad de concentración de azúcares fermentables y de compuestos organolépticos terminales de la ruta de los fenilpropanoides (Pastenes, 2007).

\section{Masa fresca}

La masa fresca de los frutos presentó diferencias significativas a nivel de tratamientos $(P \leq 0,01)$, durante los estadios herbáceo, envero, maduración y vendimia, la mayor fuente para ellos fue la hoja 6, lográndose una acumulación de biomasa fresca de 0,$92 ; 0,97 ; 1,34$ y 1,67 g respectivamente; durante la vendimia la menor 


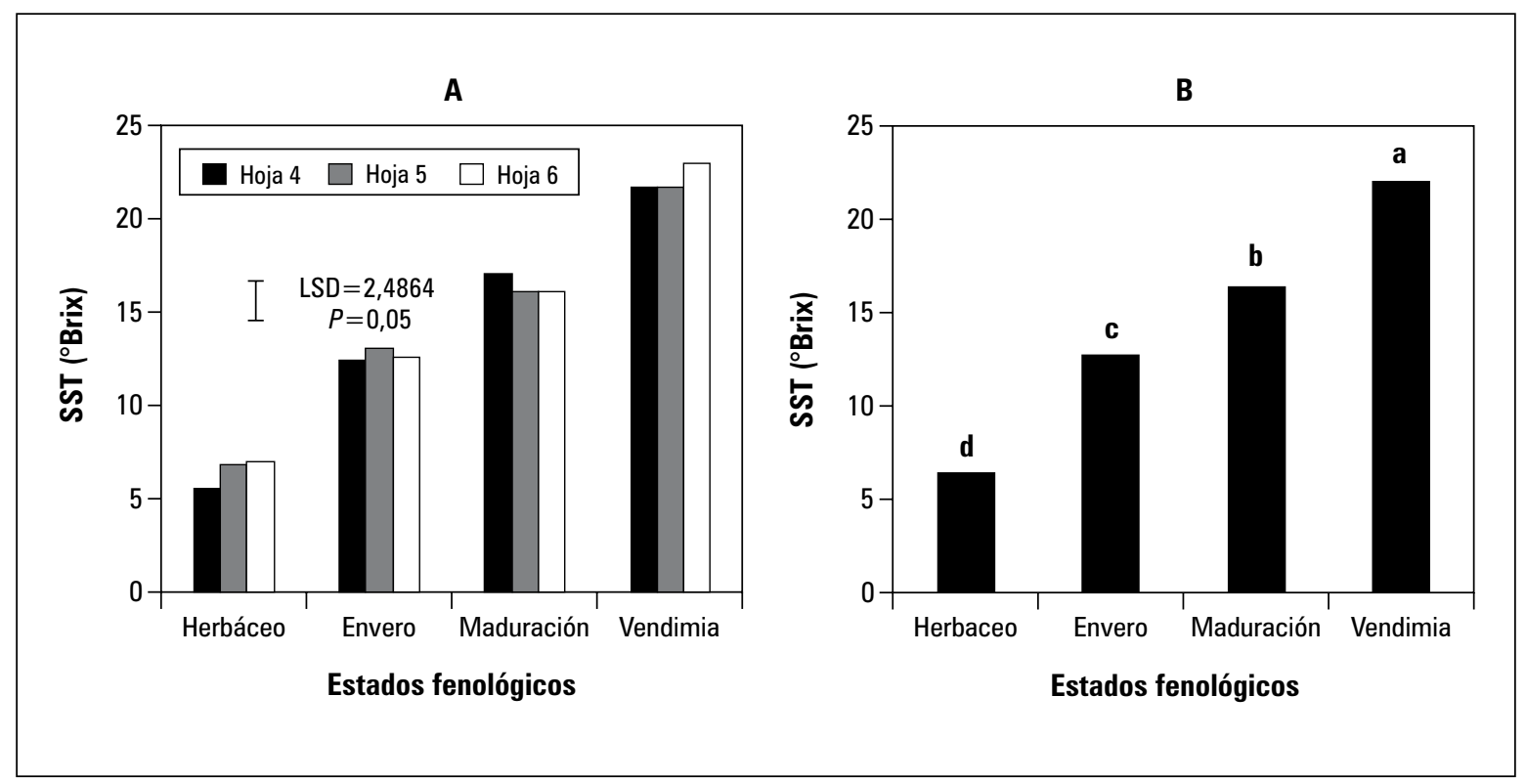

Figura 1. Contenido de sólidos solubles totales (SST, ${ }^{\circ}$ Brix) de frutos de uva de acuerdo con el estado fenológico y la ubicación de la hoja. A. Efecto de tratamientos, B. Efecto del estado fenológico. La barra vertical en A indica el valor estadístico de diferencia mínima significativa (LSD) de la prueba de Tukey. Si la diferencia entre dos promedios es superior al LSD, hay diferencias estadísticas $(\alpha=0,05)$. En $B$, promedios con letras distintas indican diferencia significativa según la prueba de Tukey $(\alpha=0,05)$.

acumulación, se mostró con el tratamiento de la hoja 4 , en donde se alcanzó un valor de 1,608 g (figura 2A).

Es así como al finalizar el estadio de maduración de los frutos de la vid las mayores fuentes son las hojas superiores, por ser estas las de mayor actividad fotosintética, esta actividad es función de una serie de factores ambientales, esta también depende de la magnitud y de la naturaleza de los órganos demandantes (Evans, 1996) y del estado nutricional de las plantas (Costa et al., 2006; Carvalho et al., 2007); en tanto que las hojas basales son las que contribuyen esencialmente, para la nutrición del tallo, tronco y raíz; convirtiéndose en los principales aportantes para asegurar la acumulación de reservas que serán las responsables de la siguiente cosecha (Motomura, 1990; Martínez de Toda, 1991). Durante los estadios fenológicos herbáceo y envero, los fotosintatos producidos por las hojas basales y medias del pámpano son destinados mayoritariamente al desarrollo de las bayas, mientras que las hojas apicales utilizan la producción de su fotosíntesis para completar el crecimiento vegetativo del brote y de su propia área foliar (Balcar y Hernández, 1988). En consecuencia, la actividad fotosintética de los segmentos basal y medio se habría visto estimulada por la demanda de fotoasimilados destinados al crecimiento y desarrollo de las bayas, pues se sabe que la presencia de frutos estimula la fotosíntesis en la vid (lacono et al., 1995).

La posición de la hoja no tuvo efecto significativo. Sin embargo, el mayor aporte en masa fresca hacia el fruto lo hizo la hoja 6 con $0,706 \mathrm{~g}$ y el menor la hoja 5 con 0,703 g. Por su parte, el estadio fenológico generó diferencias significativas en la masa fresca de frutos $(P \leq 0,01)$, el aumento más representativo se presentó durante el estadio herbáceo con un incremento de $0,68 \mathrm{~g}$, pasando de 0,28 hasta $0,96 \mathrm{~g}$ al inicio del envero, el menor incremento se presentó desde el inicio de maduración (1,33 g) hasta 1,64 g en vendimia, incrementándose 0,31 g durante esta etapa (figura 
2B). Esto concuerda con lo encontrado por Hernández (2000) quien manifiesta que el mayor índice de incremento del fruto de uva se presenta durante la primer fase de la etapa de crecimiento $y$ desarrollo, en donde se presenta una alta división y expansión celular, debido a ello, se confirma que a medida que las hojas adquieren mayor desarrollo y especialización se convierten en las principales fuentes, convirtiéndose el fruto como el mayor demandante de fotosintatos, durante los periodos herbáceo y envero (Almanza, 2012). Intrieri et al. (1992) encontraron que en hojas de vid con 140 días de edad mantienen el 70\% de la tasa máxima fotosintética, debido a que los cambios en la acumulación de clorofila, están asociados con cambios anatómicos y fisiológicos de las hojas en su desarrollo.

\section{Masa seca}

La posición de la hoja no generó diferencias significativas en la masa seca de los frutos. Sin embargo, la hoja que aportó mayor contenido fue la ubicada en el nudo número 6 con 0,0796 g y la de menor aporte fue la hoja 5 con 0,0792 g. Respecto a los estadios fenológicos, se observó un incremento de la masa seca en función del desarrollo de los frutos $(P \leq 0,01)$ alcanzando un valor de $0,177 \mathrm{~g}$ en la vendimia (figura $3 \mathrm{~B}$ ).

Los tratamientos tuvieron efecto significativo y la mayor masa seca de fruto se obtuvo con la hoja 5 en la vendimia (figura 3A). Del estadio herbáceo del fruto al estadio envero se logró un alto porcentaje de acumulación de masa seca. Mientras que el aumento durante la maduración a vendimia fue más lento; la hoja que realizó mayores aportes fue la 4 (0,154 g), en tanto que en la vendimia el aporte máximo de masa seca al fruto, lo hizo la hoja 5 con $0,1776 \mathrm{~g}$ (figura $3 \mathrm{~A}$ ), queda demostrado que a medida que avanza el crecimiento y desarrollo de las hojas y por tanto el desarrollo fenológico, las hojas adultas van perdiendo actividad metabólica, y además, se corrobora lo mencionado por Hunter y Visser (1988), quienes mencionan que los principales

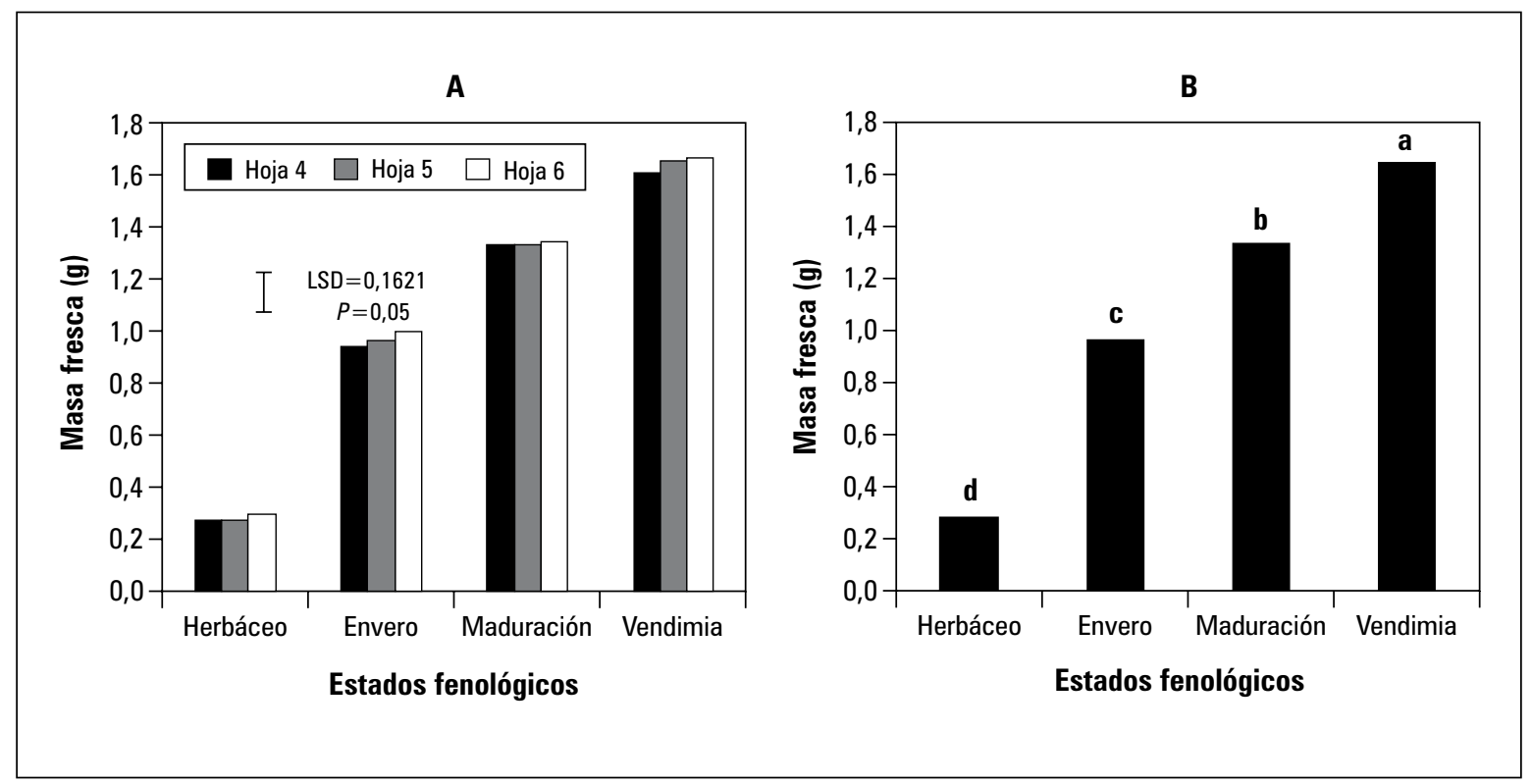

Figura 2. Masa fresca de frutos de uva de acuerdo con el estado fenológico y la ubicación de la hoja. A. Efecto de tratamientos, B. Efecto del estado fenológico. La barra vertical en A indica el valor estadístico de diferencia mínima significativa (LSD) de la prueba de Tukey. Si la diferencia entre dos promedios es superior al LSD, hay diferencias estadísticas $(\alpha=0,05)$. En $B$, promedios con letras distintas indican diferencia significativa según la prueba de Tukey $(\alpha=0,05)$. 
sumideros después del cuajado del fruto son las propias bayas y que dependiendo de la posición de la hoja en el sarmiento, se distribuyen los fotosin- tatos hacia vertederos más fuertes, que sería el caso de la hoja 4 en donde su distribución se realizaría hacia el tronco y raíces (Martínez de Toda, 1991).

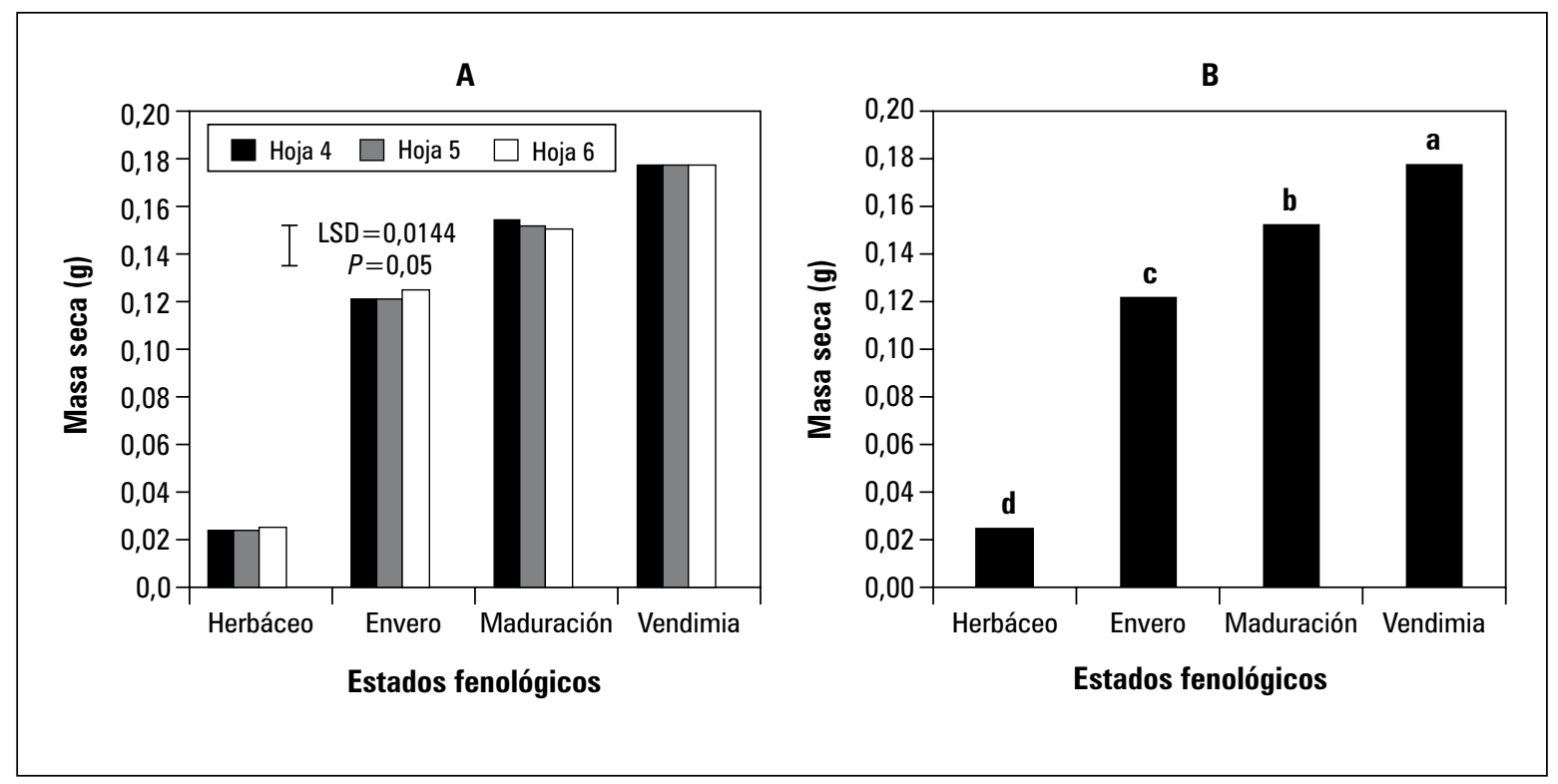

Figura 3. Masa seca de frutos de uva de acuerdo con el estado fenológico y la ubicación de la hoja. A. Efecto de tratamientos, B. Efecto del estado fenológico. La barra vertical en A indica el valor estadístico de diferencia mínima significativa (LSD) de la prueba de Tukey. Si la diferencia entre dos promedios es superior al LSD, hay diferencias estadísticas $(\alpha=0,05)$. En $B$, promedios con letras distintas indican diferencia significativa según la prueba de Tukey $(\alpha=0,05)$.

\section{CONCLUSIONES}

Bajo las condiciones del estudio se encontró, que a medida que los frutos de uva de la selección clonal 'Riesling x Silvaner' incrementan su de- sarrollo, los sólidos solubles totales, la masa fresca y la masa seca de las bayas se hace mayor en la vendimia. Además, la hoja 6 en la vendimia generó mayor concentración de sólidos solubles totales y masa fresca de bayas.

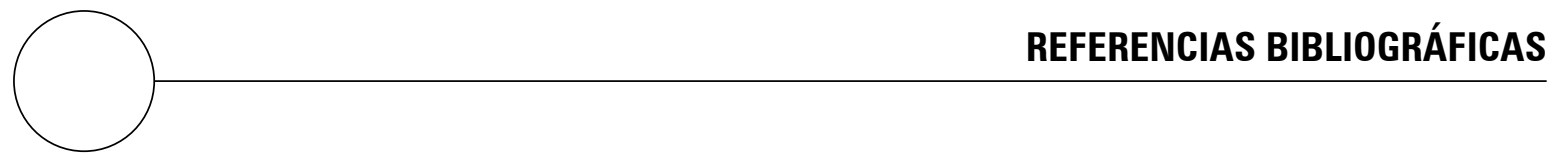

Agronet, 2012. Uva. En: http://agronet.gov.co; consulta: enero de 2012.

Ali, K., F. Maltese, A.M. Fortes, M.S. Pais, Y.H. Choi y R. Verpoorte. 2011. Monitoring biochemical changes during grape berry development in Portuguese cultivars by NMR spectroscopy. Food Chem. 124, 1760-1769.
Alleweldt, G., R, Eibach y E. Rühl. 1982. Untersuchungen zum Gswechsel der Rebe. I. Einfluß von Temperatur, Blattalter und Tageszeit auf Nettophotosynthese und Transpiration. Vitis 21, 93-100.

Almanza, P.J. 2012. Evolución de la clorofila foliar durante el ciclo reproductor de la vid (Vitis vinifera L.) Trabajo para ascenso en el escalafón docente. 
Facultad de Ciencias Agropecuarias, Universidad Pedagógica y Tecnológica de Colombia, Tunja.

Almanza, P.J. 2011. Determinación del crecimiento y desarrollo del fruto de vid (Vitis vinifera L.) bajo condiciones de clima frío tropical. Tesis de doctorado. Facultad de Agronomía, Universidad Nacional de Colombia, Bogotá.

Balcar, J. y J. Hernández. 1988. Translocación de fotosintatos en sarmientos de la vid durante el periodo vegetativo. Vitis 27, 13-20.

Buttrose, M.S. 1966. The effect of reducing leaf area on the growth of roots, stems and berries of Gordo grapevines. Vitis 5, 455-464.

Candolfi-Vasconcelos, M.C., W. Koblet, G. Howell y W. Zweifel. 1994. Influence of defoliation, rootstock, training system and leaf position on gas exchange of Pinot noir grapevines. Amer. J. Enol. Vitic. 45(2), 173-180.

Conde, C., P. Silva, N. Fontes, A.C.P. Dias, R.M. Tavares, M.J. Sousa, A. Agasse, S. Delrot y H. Gerós. 2007. Biochemical changes throughout grape berry development and fruit and wine quality. Food 1(1), 1-22.

Costa, K.A., I. Oliveira. y V. Faquin. 2006. Concentrações de nitrogênio e estimativa do teor de clorofila. En: De Oliveira, M.A.S. (ed.). Adubação nitrogenada para pastagens do gênero Brachiaria em solos do Cerrado. Embrapa Arroz e Feijão, Santo Antônio de Goiás, GO, Brasil.

Carvalho, L., P. Bonomo, J.A. Dos Santos, F.M. De Jesus, A. Diaz y A.J. Vieira. 2007. Concentração de nitrogênio em folhas de dois cultivares de braquiária através de leitura com o clorofilômetro. Revista Electrónica de Veterinaria 8(1).

Evans, L. 1996. Crop, evolution, adaptation and yield. Cambridge University Press, Cambridge, Londres.

Fillion, L., A. Ageorges, S. Picaud, P. Coutos-Thevenot, R. Lemoine, C. Romieu y S. Delrot. 1999. Cloning and expression of a hexose transporter gene expressed during the ripening of grape berry. Plant Physiol. 120, 1083-1093.

Hidalgo, L. 1999. Tratado de viticultura general. $2^{\text {a }}$ ed. Ediciones Mundi-Prensa, Madrid.

Hernández, J., I. Escobar y N. Castilla. 2001. La radiación solar en invernaderos mediterráneos. Horticultura global: Revista de Industria, Distribución y Socioeconomía Hortícola 157, 18-27.

Hernández, A. 2000. Introducción al vino de Chile. Colección en Agricultura de la Facultad de Agronomía e Ingeniería forestal. Pontifica Universidad Católica de Chile, Santiago.

Hunter, J. y E. Archer. 2002. Papel actual y perspectivas futuras de la gestión del follaje. AC revista de enología No. 21. En: http//acenología.com/ ciencia59_2htm; consulta: abril de 2012.

Hunter, J.J. 2000. Implications of seasonal canopy management and growth compensation in grapevine. S. Afr. J. Enol. Vitic. 21, 81-91.

Hunter, J.J. y J.H. Visser, 1988. Distribution of ${ }^{14} \mathrm{C}$ photosynthetate in the shoot of Vitis vinifera L. cv. Cabernet Sauvignon. I. The effect of leaf position and developmental stage of the vine. S. Afr. J. Enol. Vitic. 9(2), 3-9.

Hunter, J.J., H.P. Ruffner, C.G. Volschenk y D.J. Leroux. 1995. Partial defoliation of Vitis vinifera L. cv. Cabernet-Sauvignon/99 Richter: effect on root growth, canopy efficiency, grape composition and wine quality. Amer. J. Enol. Vitic. 46(3), 306-314.

Iacono, F., M. Bertamini, A. Scienza y B. Coombe. 1995. Differential effects of canopy manipulation and shading of Vitis vinifera L. cv. Cabernet Sauvignon. Leaf gas exchange, photosynthetic electron transport rate and sugar accumulation in berries. Vitis 34(4), 201- 206.

Intrieri, C., S. Poni, O. Silvestroni y I. Filippetti. 1992. Leaf age, leaf position and photosynthesis in potted grapevines. Adv. Hort. Sci. 1, 23-27.

Johnson, R.S. y A.N. Lakso. 1985. Relationships between stem length, leaf area, stem weight, and accumulated growing degree-days in apple shoots. J. Amer. Soc. Hortic. Sci. 110(4), 586-590.

Katerji, N., A. Carbonneau, F.A. Daudet y N. Olat. 1994. Etude á l'échelle de la plante entiére du fontionnement hydricque et photosynthétique de la vigne: comparaison des systémes de conduite tradionnel et en Lyre. Vitis 33, 197-203.

Kliewer, W.M. 1980. Vineyard canopy management a review. pp. 342-352. En: Webb, A.D. (ed.). Proc. Grape and Wine Centennial Symp., 18-21 Junio, 1980. Davis, CA.

Klobet, W. 1969. Migration of assimilates in vine shoots and influence of the leaf surface on grape yield and quality. Wein-Wissensch. 24, 277-319.

Lorenz, D.H., K.W. Eichhorn, H. Bleiholder, R. Klose, U. Maier y E. Weber. 1995. Phenological growth stag- 
es of the grapevine (Vitis vinifera L. ssp. vinifera)Codes and descriptions according to the extended BBCH scale. Aust. J. Grape Wine Res. 1, 100-103.

Mabrouk, H., A. Carbonneau y H. Sinoquet. 1997. Canopy structure and radiation regime in grapevine I. Spatial and angular distribution of leaf area in two canopy systems. Vitis 36(6), 119-123.

Martínez de Toda, F. 1991. Biología de la vid. Fundamentos biológicos de la viticultura. Ediciones MundiPrensa, Madrid.

Motomura, Y. 1990. Distribution of ${ }^{14} \mathrm{C}$-assimilates from individual leaves on clusters in grape shorts. Amer. J. Enol. Viticult. 41(4), 306-312.

Oliveira, M. y M. Santos. 1995. A semi- empirical method to estimate canopy leaf area of vineyards. Amer. J. Enol. Vitic. 46(3), 389-391.

Pastenes, C. 2007. Fotosíntesis en vides de interés enológico. Facultad de Ciencias Agronómicas. Universidad de Chile. En: htpp://www.gie.uchile.cl/ cpastene0105.pdf; consulta: mayo de 2012.

Quijano R., M. 2001. Los vinos del Valle del Sol. Cultura Científica 1, 5-11.

Rajapakse, N.C. y Y. Shahak. 2007. Light-quality manipulation by horticulture industry. pp. 290-312. En: Whitelam, G.C. y K.J. Halliday (eds.). Light and plant development. Blackwell Publishing, Oxford, UK.

Sánchez de Miguel, P. 2007. Producción y distribución de fotoasimilados en la vid (Vitis vinifera L.) durante el periodo de maduración. Cambios en la respuesta fotosintética a la luz de las hojas por factores biológicos ambientales y culturales. Tesis de doctorado. Escuela Técnica Superior de Ingenieros Agrónomos, Universidad Politécnica de Madrid, Madrid.

Schultz, H.R. 1995. Grape canopy structure, light microclimate and photosynthesis. I. A two dimensional model of the spatial distribution of surface area densities and leaf ages in two canopy systems. Vitis 34(4), 211-215.

Seleznyova, A.N. y D.H. Greer. 2001. Efects of temperature and leaf position on leaf area expansion of kiwifruit (Actinidia deliciosa) shoots: Development of a modelling framework. Ann. Bot. 88, 605-615.

Smart, R. y S. Smith. 1988. Canopy management: Identifying the problems and practical solutions. pp. 109-115. En: Proc. 2 ${ }^{\text {nd }}$ International Cool Climate Viticulture and Oenology Symposium. Auckland, New Zealand.

Stewart, W.P., B.M. Freeman y J.K. Dick. 1996. Carbon partitioning in potted post- veraison Vitis vinifera L. cv. Riesling vines: Effect of dormant cane length. S. Afr. J. Enol.Vitic. 17(2), 38-42.

Vasconcelos, M.C. y S. Castagnoli. 2000. Leaf canopy structure and vine performance. Amer. J. Enol. Vitic. 51(4), 390-396.

Zufferey, V., F. Murisier y R. Schultz. 2000. A model analysis of the photosynthetic response of Vitis vinifera L. cvs. Riesling and Chasselas leaves in the field: I. Interaction of age, light and temperature. Vitis 39(1), 19-26. 